\title{
Real-Time Monitoring of Charge Accumulation from Insole-Mounted MFC Piezoelectric Modules for Analysis of Power Availability in Mobility-Restricted Patients
}

\author{
Sian Armstrong, Cathy Holt, Philippa Jones \\ School of Engineering, Cardiff University \\ The Parade, Cardiff, Wales, UK \\ Armstrongs2@cf.ac.uk; Holt@cf.ac.uk; Jonesp29@cf.ac.uk
}

\section{Extended Abstract}

Almost 10 million people suffer from musculoskeletal conditions in the UK alone, causing pain and reduced mobility, with knock-on impact to medical consultations and associated cost, ability to work, mental health and reduced quality of life. Collection of biomechanical data between clinics would allow optimisation of treatment, improving patient outcomes and pain scores. Advances in telemedicine and miniaturised, low-power wireless wearables could be exploited to better understand patient mobility away from the clinic, and inform treatment regimes.

A self-powered active monitoring shoe insole would enable such data to be collected, and remotely monitored and analysed, with no intervention from the patient.

Monitoring activity at the foot both affords a level of human-generated power not available elsewhere upon the body [1], and an ideal location for monitoring pressure distribution, joint off-loading and temporal gait characteristics. Piezoelectric Macro Fiber Composite (MFC) materials provide a low-profile and flexible form-factor ideal for embedding into shoe insoles to exploit power generated during walking [2, 3]. Likewise, flat and flexible force-sensing resistive sensors, combined with miniaturised MEMS accelerometers can provide data with proven application to gait analysis [4].

A challenge to power generation in musculoskeletal patients however is that mobility-restricted users would produce inherently lower levels of power due to reduced levels of activity. The experimentation reported in this paper determines first a baseline of power generation for different rates of walking for MFC modules placed at the heel and forefoot within the insole in able-bodied participants. Accumulation of charge for different activities of daily living expected of those with motion-restricting musculoskeletal conditions will then be presented, collected from a cohort of participants noting their height and weight. Such activities include: walking at a comfortable speed over short distances, walking up and down short flights of stairs, sitting to standing, standing to sitting, getting in and out of bed, and other typical activities.

Experimental set up comprises an insole fitted with two MFC modules measuring $85 \times 57 \mathrm{~mm}$ in the forefoot and $56 \times 28 \mathrm{~mm}$ in the heel of the insole, with charge accumulation data collected wirelessly using a Bluetooth-enabled wireless sensor node to a laptop. The wireless node additionally monitors accelerometer data for accurate comparison of charge generation against different rates of walking and to better understand the activities' charge-generating capabilities. Additionally motion capture and accelerometer data (collected using gold standard facilities at Cardiff's Musculoskeletal Biomechanics Research Facility (MSKBRF) which includes motion capture, force plates and instrumented treadmill) will also be used to determine a lower boundary for sampling rate of sensors to yield useful biomechanical data of the activities of interest.

State of the art ultra-low power microcontrollers and sensing circuitry will be compared to present ranges of power consumption for different levels of data collection activity. This will allow proposals for optimum sampling rate in order to yield appropriate activity data without exceeding available power levels, and methods for adapting both number of active sensors and their sampling rate based upon current level of patient activity, to ensure energy demand does not exceed supply. A proposal for power-optimal upload of data for Telehealth monitoring in a practical home scenario will also be presented. 


\section{References}

[1] T. Starner, "Human-powered wearable computing," IBM Systems Journal, vol. 35, no. 3.4, pp. 618-629, 1996.

[2] J. A. Paradiso and T. Starner, "Energy scavenging for mobile and wireless electronics," IEEE Pervasive Computing, vol. 4, no. 1, pp. 18-27, 2005.

[3] R. Meier, N. Kelly, O. Almog, and P. Chiang, "A piezoelectric energy-harvesting shoe system for podiatric sensing," in 2014 36th Annual International Conference of the IEEE Engineering in Medicine and Biology Society, 2014, pp. $622-625$.

[4] W. Tao, T. Liu, R. Zheng, and H. Feng, "Gait Analysis Using Wearable Sensors," Sensors, vol. 12, no. $2,2012$. 